\title{
Multilayer Traffic Engineering in Interworking Multihop wireless networks
}

\author{
Oladayo Bello \\ School of Information Technology, Monash University, South Africa campus, \\ Ruimsig, South Africa. \\ E-mail: oladayo@ieee.org
}

Antoine Bagula

ISAT Laboratory, Department of Computer Science, University of Cape Town,

Cape Town, South Africa

E-mail: bagula@cs.uct.ac.za

H.Anthony Chan

Huawei Technologies, Core Network Research, Texas, USA.

Email: h.a.chan@ieee.org

Received: January 8, 2012

DOI: $10.5296 /$ npa.v4i2.1264
Accepted: May 15, 2012 Published: June 19, 2012

URL: http://dx.doi.org/10.5296/npa.v4i2.1264

\begin{abstract}
The advancement in wireless networking and wireless device technology are paving the way for bringing the vision of ubiquitous communication to reality. This vision will be enabled by the interworking of existing wireless multihop wireless networks. However, the diversity in the design and operation of these wireless networks may not enable users to enjoy continuous network service as they traverse between networks. This paper presents a framework which ensures continuous service and sustains an acceptable service level quality for network users transiting between multiple multihop wireless networks. The focus is on the scenario where a user utilizes multiple hops to access any of the multiple networks. We classify the components of the framework into link discovery, resource optimization and routing
\end{abstract}


processes. A set of analytical models and metrics are defined for these processes and the framework evaluated with simulations. The findings show that despite an increase in simultaneous network users, which degrades network performance, the framework is able to support quality continuous service for users transiting between networks.

Keywords: Interworking, Multihop, Wireless network, Traffic Engineering.

\section{Introduction}

The emergence of different types of network services (e.g. IPTV, video on demand etc), an increase in the demand for these services and most especially the desire for "more convenient" ways to access these services have led to the evolution of different wireless access networks. Over the years, several wireless networks (fixed/mobile networks, single-hop/multihop mobile networks, infrastructure-based/infrastructure-less networks) have been designed with more sophisticated standards than their predecessors in order to satisfy the demand cravings. Though these wireless access network technologies have been standardized and commercialized, yet no single technology can be considered as the best because they have been designed for different purposes and therefore operate with different networking standards [1]. In addition, there is a slow upgrade and migration to new wireless technologies due to the involved cost.

Wireless access networks typically have distinguishing characteristics in terms of coverage range, frequency of operation, data rate and power [2]. With the increase in demand for seamless service continuity through ubiquitous broadband connectivity, the integration of multiple heterogeneous wireless access networks has become important [1]. Heterogeneity is supported with the fact that no single wireless network standard is able to optimally cover all the different wireless communication scenarios [3] [4]. The integration of wireless networks can allow the provisioning of services to mobile network users in any communication scenario.

In order to achieve the integration of wireless networks, the networks are seamlessly inter-networked (interworked). The concept of inter-working networks does not create an entirely new network; it is just an approach that enables the integration of existing network technologies into a unified platform in order to provide an uninterrupted and consistent level of service to network users. Moreover, to complement the quest for access to services anytime and anywhere, mobile devices are now being equipped with multi-mode (multi-technology) capabilities, which can allow users to have access to any available network using a single mobile device. Therefore, apart from the integration of wireless networks, there is also a tendency that heterogeneous terminals (nodes) can co-exist in an interworking wireless network.

This paper proposes a multilayer traffic engineering framework to ensure ubiquitous connectivity for user in interworking multihop wireless networks as they transit between networks. The analytical probabilistic models that make up the framework and the simulation performance of the framework are presented. The paper is organized as follows: Section 2 
provides a review of interworking wireless networks. Section 3 explains the challenges of traffic engineering in interworking multihop wireless networks. Section 4 illustrates the deployment scenario considered in this research. Sections 5 and 6 present the analysis of the link availability and non-impairment probability models. The connectivity model is presented in section 7 while section 8 analyzes the simulation performance of the framework.

\section{Review of interworking wireless networks}

A lot of research work has gone into the interworking of single-hop WWAN (e.g. 3G) and single-hop WLAN (e.g. IEEE802.11). Standardization groups such as the Third Generation Partnership Project (3GPP) [5] and 3GPP2 [6] have dealt with the interworking of $3 \mathrm{G}$ and WLANs. The interworking of heterogeneous IEEE 802 networks and between IEEE 802 networks and cellular networks has also been dealt with by standardization groups. However, they have only considered the single-hop mode of operation, where only two connection options are available. These options are: 1) direct connection between a mobile terminal and a cellular Base Station (BS) or 2) direct connection between a mobile terminal and an Access Point (AP). The paradigm of multihop communication was not exploited. Even though coverage extension and relaying via multihop communication is an old concept, it has become practical only recently [7]. Practitioners expect that in the future, wireless networks will not be limited to cellular systems [8]. It is envisaged that parts of the access domain in next generation wireless networks will not be centrally organized, instead they will be infrastructure-less and provide multihop communication for nodes that cannot reach their destination with a single hop transmission [9]. Mobile terminals (nodes) may access network services by forming alliance with other nodes any time and any place. Such nodes could be a part of the personal, local and wide area sphere of wireless networking. The nodes would temporarily co-operate to provide ubiquitous access and service continuity for each other [9]. In addition, to reduce the problem of dead spot in cellular networks, multihop cellular networks are also being considered [10].

The advent of multihop communications has brought about new opportunities for future wireless networks (e.g. seamless service continuity) [11]. In contrast to single hop, multihop capabilities allow nodes to relay traffic for each other. It introduces the concept of cooperation among nodes to allow short-range communication between them. It also achieves higher spectral efficiency, better route diversity, and significantly lowers power consumption. In addition, it alleviates path loss and fading issues associated with the wireless channel. Mitigating path loss and fading, and the reduction of consumed power arise from the ability of nodes to provide each other with diverse propagation paths through a series of close hops to intended destination [12]. For example, splitting a single-hop connection into two hops reduces the average distance between communicating pairs of nodes. Since power consumption is proportional to distance, the reduction in distance results in the reduction of the amount of energy that would have been used to transmit the data on a single-hop. In this way, multihop wireless networks are able to improve the reliability of the communication between nodes and extend the network capacity [12]. In addition, because there can be 
diverse multihop routes to a destination, multihop communications introduce inherent redundancy, which upholds the network during random link failure. With all the advantages of multihop communication, some drawbacks do exist. One drawback is an increase in the amount of traffic that will be handled by nodes which are closer to the base station. However, in order to provide good coverage in a large network, multihop communication is inevitable as the advantages outweigh the drawbacks.

Some applications of multihop communication include mesh networks, vehicular networks, and mobile ad-hoc and sensor networks. Furthermore, the recent enhancements to wireless network technologies such WiFi (Wireless Fidelity), WiMAX (Worldwide Interoperability for Microwave Access) and cellular networks include the addition of multihop capability. These enhanced standards are the IEEE 802.16j standards for mesh and multihop relay specification in Mobile WiMAX [7] [13], the 3rd Generation Partnership Project (3GPP) Opportunistic Driven Multiple Access (ODMA) which is a protocol proposed for UMTS TDD mode [14] [15], the IEEE 802.11s which is an ESS (Extended Service Set) mesh networking enhancement of IEEE 802.11, and the IEEE 802.15.5 for WPAN (Wireless Personal Area Network) mesh networking [16].

For the provisioning of ubiquitous service access, extended coverage, increased data rate and seamless service continuity, the interworking of multihop wireless networks (integration of multihop wireless networks) is beneficial. Integrated architectures such as single-hop WWAN and single-hop WLAN, and single-hop WWAN/WLAN and multihop WLAN/WWAN only enables a user to access a particular network depending upon the application needs and the available networks. On the other hand, the integration of heterogeneous multihop wireless networks provides more connectivity options and unlimited mobility. Tables 1, 2 and 3 outlines the connection options that may be available for single-mode and multi-mode nodes in the three integrated architectures.

Table 1. Connection options in an integrated single-hop WWAN and single-hop WLAN architecture

\begin{tabular}{|l|c|c|}
\hline Access nodes & WWAN & WLAN \\
\hline Single-mode WLAN & $\square$ & $\square$ \\
\hline Single-mode WWAN & $\square$ & $\square$ \\
\hline Multi-mode node & $\square$ & $\square$ \\
\hline
\end{tabular}

From table 1, single-mode nodes can only have access to their services through appropriate gateway nodes; however, multi-mode nodes can have access via any available access node. 
Table 2. Connection options in an integrated single-hop WWAN and multihop WLAN architecture

\begin{tabular}{|l|c|c|c|}
\hline \multicolumn{1}{|c|}{ Access nodes } & WWAN & WLAN & Multihop \\
\hline User nodes & $\nabla$ & $\square$ & $\square$ \\
\hline Single mode WLAN & $\square$ & $\square$ & $\square$ \\
\hline Multi-mode node & $\square$ & $\square$ & $\square$ \\
\hline
\end{tabular}

Comparing table 1 with table 2, multihop nodes further increase network service access for users in networks integrating single-hop WWAN and multihop WLAN.

Table 3. Connection options in an integrated multihop WWAN and multihop WLAN network

\begin{tabular}{|l|c|c|c|c|c|}
\hline User nodes & WWAN & WLAN & $\begin{array}{c}\text { Multihop } \\
\text { single-mode }\end{array}$ & $\begin{array}{c}\text { Multihop } \\
\text { single-mode }\end{array}$ & $\begin{array}{c}\text { Multihop } \\
\text { Multi-mode }\end{array}$ \\
\hline Single mode WLAN & $\square$ & $\square$ & $\square$ & $\square$ & $\square$ \\
\hline Single-mode WWAN & $\square$ & $\square$ & $\square$ & $\square$ & $\square$ \\
\hline $\begin{array}{l}\text { Multi-mode node (WLAN } \\
\text { and WWAN) }\end{array}$ & $\square$ & $\square$ & $\square$ & $\square$ & $\square$ \\
\hline
\end{tabular}

Comparing table 1, table 2 and table 3, integrated multihop WWAN and WLAN provides more options for service access and thus allows ubiquitous and seamless service continuity. Note that the provisioning of ubiquitous access and seamless service continuity are not dependent only on inter-working mechanisms but also on the traffic engineering protocols used by the networks involved.

Therefore, in the light of the provisioning of ubiquitous network access to users, the interworking of multihop wireless networks is a step into the future of wireless networking. The interworking of multihop wireless networks can substantially extend network coverage and provide unlimited and ubiquitous network access so that users can enjoy seamless handover and uninterrupted service. These networks can also be very useful in areas that are 'hard to wire', areas with no initial wire-line or wireless single hop network coverage and areas that suffer from connectivity problems e.g. isolated inhabited islands or rural areas. They are also useful for military applications and communication restoration in disaster recovery operations when cell sites may have been damaged. Another application is for Intelligent Transport Systems, which ensures road safety. In addition, the interworking of multihop wireless networks can minimize infrastructure cost and thus provide low-cost networking.

Despite the advantages and potentials that come with the interworking of multihop 
wireless networks, the inter-working process poses many challenges. The research issues in interworking multihop wireless networks covers all layers of the network protocol stack. Some of these research issues are related to traffic engineering, mobility, billing and security [17]. However, this research specifically focuses on traffic engineering in inter-working multihop wireless networks in which heterogeneous mobile terminals co-exist. Since mobile terminals are now being equipped to integrate different access technologies on a single mobile device, the co-existence of single-mode and multi-mode mobile devices in a network cannot be undermined. Integrating multiple technologies on a single device allows network users to be supported by any available network. Thus, the issue of resource optimization still exists in the inter-working of multihop wireless networks. This research considers a case where all terminals (single-mode or multi-mode) have multihop capability.

\section{Challenges of Traffic engineering in inter-working multihop wireless networks}

Traffic engineering (TE) is a commonly-used technique for achieving optimal or near optimal performance in a network. Traffic engineering concepts allow a network to exercise control over the way it provides or responds to demand for resources by moving the traffic offered to a network to where the network resources are available. The control is achieved with the use of protocols that influence the manner in which resources may be allocated. TE can be viewed in different ways depending upon the specific context in which they are used and the goal they serve. The optimization objective of TE can be achieved through capacity management or traffic management. Capacity management includes capacity control, routing control, and resource management. Traffic management includes nodal traffic control functions such as traffic conditioning, queue management and scheduling [18]. The manner in which traffic engineering objectives are implemented change over time as new requirements are imposed by emerging technologies. Thus, new TE methods/concepts are required to enhance network performance as new technologies emerge. Due to the nature of wireless networks, designing TE mechanisms for wireless networks is more challenging than for wired networks [19].

Fig. 1 shows the three main steps of a traffic engineering mechanism. The first step is the acquisition of knowledge about underlying network metrics and associated resource constraints. Second is an optimization of the network performance measure of interest. The performance measures of a network may be traffic level QoS parameters such as throughput, packet loss, delay and jitter. The last step is the routing of traffic through paths that optimize the necessary performance measures.

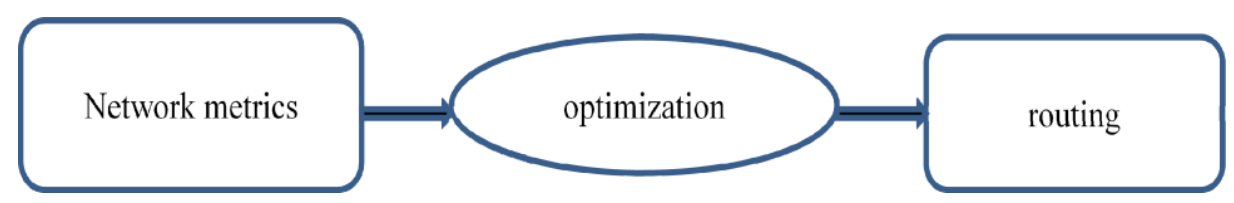

Figure 1 Steps in traffic engineering processes [20] 
Traffic engineering functions are vital in ensuring ubiquitous access and service continuity for nodes (mobile terminals) in an inter-working multihop wireless network. Two features of such networks that significantly affect TE are the co-existence of single and multi-modal terminals and the multihop communication capability of the nodes.

Typically, in a wireless network, all nodes run the same set of protocols (e.g. on the lower layers: physical, link and MAC layer) and these protocols have the same goal when providing services to the upper layers. However, with the co-existence of single-mode and multi-mode nodes, nodes may implement different protocols for a given layer (e.g. physical and MAC layer protocols may differ). Thus, inter-working may cause the operation of the protocols to conflict. For example, if different scheduling techniques are employed by the MAC protocols, the concurrent operation of these techniques in an inter-worked scenario may eventually cause inter-node interference. This occurrence may significantly affect the traffic engineering process within the network and must be taken into account [17]. In order to leverage the capabilities of inter-working multihop wireless networks to provide seamless service continuity and ubiquitous broadband access, appropriate traffic engineering mechanisms are needed.

Due to the multihop communication capability within the network, the identification of the optimal and reliable paths for traffic routing is very important. Traffic routing is mainly affected by connectivity constraint within the network. In an inter-working multihop wireless network, node to node connections may involve a sequence of several links and the final end-to-end connection experienced by a network user will be limited by the weakest link in this chain of connections. Thus, TE techniques should select paths that will optimize connectivity and avoid the wastage of network's resources while ensuring ubiquitous access and service continuity. The dynamic network topology and the random location of nodes make it difficult to discover and maintain reliable routes. In addition, end-to-end delay can occur due to problems at the lower layers (e.g. interference patterns on the physical layer). Hence, is it necessary to consider the operations on the lower layers in traffic engineering.

The multi-layer traffic engineering framework presented in this paper consists of a link discovery optimization process, a resource optimization process and a routing process. The framework encompasses metrics on the physical and MAC layer that affects traffic engineering in inter-working multihop wireless networks. We disintegrate the traffic engineering problem by looking into the issue of connectivity in inter-working multihop wireless networks, which has not been studied.

A multi-layer approach to TE in interworking multihop wireless networks will ensure the utilization of useful lower layer information in the link discovery optimization and resource optimization for the routing process. The approach will allow traffic and resource level parameters on the lower layer to be taken into account in the optimization processes. The optimization objective of the framework presented is to optimize connectivity for nodes within the network. Basically, the objective of the multi-layer approach is to optimize connectivity by choosing the link with the optimal resource through the link discovery process and resource optimization process. 
The link discovery and resource optimization processes determine the level of connectivity between two multihop nodes. Link discovery involves finding an available link for a node that wants to transmit. Resource optimization involves ensuring that an available link is highly reliable for the transmission. These processes utilize parameters such as node's transmission range, transmission power, spatial node density, interfering node density, probability of interference, signal to noise and interference ratio (SNIR) and bit error probability. During the routing process, the level of connectivity on a link is the routing cost metric. A strong connectivity level on all links that make up a multihop route means that reliable last mile ubiquitous service continuity can be assured.

In this paper, connectivity is taken as a probabilistic measure due to the stochastic nature of the wireless environment. We define connectivity as the probability that a wireless link is available and its non-impairment probability is high enough to guarantee successful transmission over it. Therefore, connectivity is mutually dependent on a link's availability and non-impairment probability. Link availability is the probability that two nodes are within at most the maximum transmission range that is sufficient for a communication link to be established between them. Non-impairment probability indicates the likelihood that the radio attributes of a link has the potential to satisfy the minimum requirement for successful communication over it. The minimum requirement is defined by the underlying network parameters and associated resource constraints of the network such as data rate and the SNIR threshold of the network. When fine-tuned, these attributes define by physical/MAC layer parameters that ensure the proper functioning of a link.

\section{Deployment Scenario}

Consider a scenario in which multihop wireless access networks are inter-worked with partially overlapped coverage as shown in fig. 2. These networks can belong to different or same service providers. Users' nodes (terminals) such as laptops and smart phones in the network are either single or multi-modal nodes with multihop capabilities. Gateway nodes (access points and base stations) provide inter-domain co-ordination between the networks as well as connection to the Internet. If single-mode nodes are out of the range of their applicable gateway, they can relay traffic through other single/multi-mode nodes. The nodes with multi-mode capability can also connect to applicable gateway directly if they are within its range, otherwise they will relay their traffic through other nodes. As nodes move, they can enjoy ubiquitous access and seamless service continuity, assuming there is co-operation between nodes. Even if nodes are out of range of their applicable gateway, their traffic can still be relayed through the closest multihop node with similar operational mode capability. When gateway nodes are unreachable by a node, the onus of all other nodes is to ensure continuity of service for this node. 


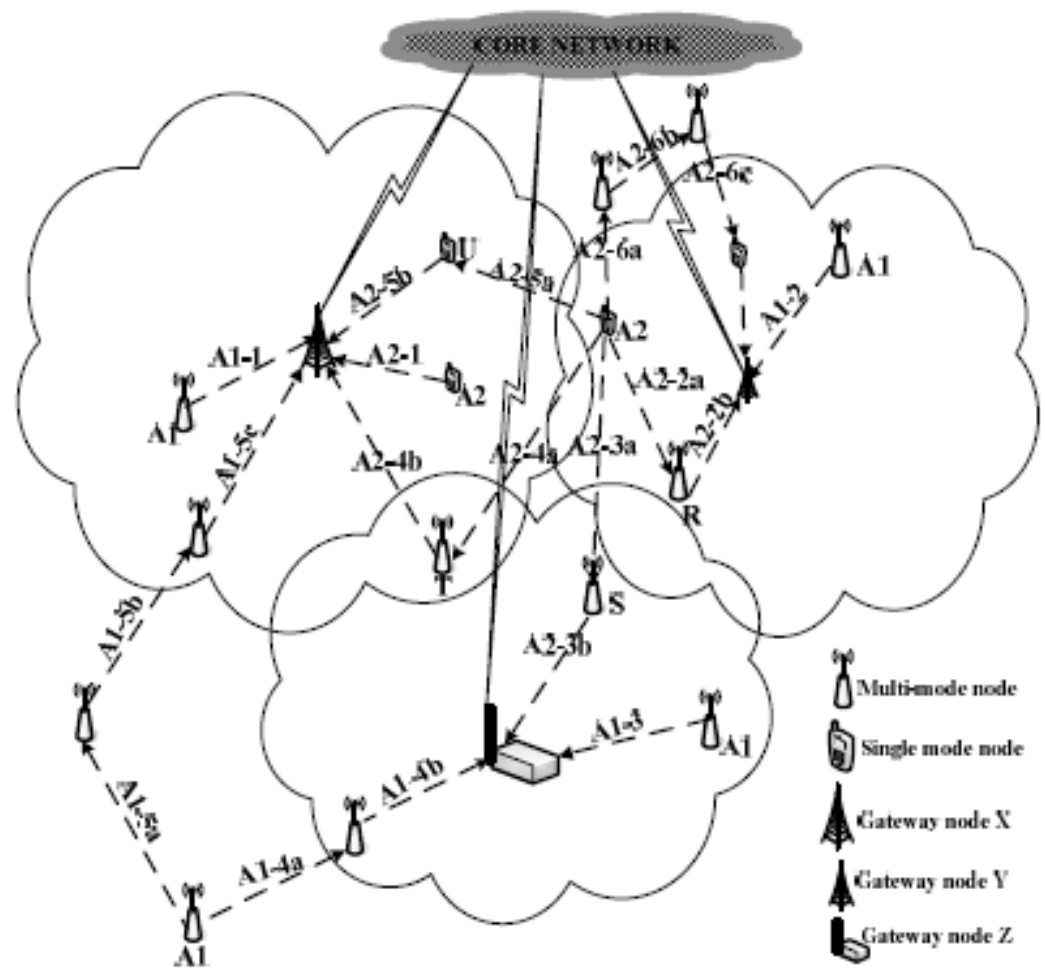

Figure 2 Inter-working network scenario with single mode and multi-mode nodes.

Fig. 2 illustrates some possible ubiquitous service continuity connections in this scenario.

From fig. 2, the communication scenarios that can be considered for node A1 (a multi-mode terminal) are:

1. It can have service continuity through gateway nodes $\mathrm{X}, \mathrm{Y}$ or $\mathrm{Z}$ if it is within their coverage range as it moves around. This is illustrated by: A1-1, A1-2 and A1-3 connections

2. If A1 happens to be out of coverage range of all the gateway nodes, it is able to continue accessing its ongoing service through either connection A1-4a and A1-4b or A1-5a, A1-5b and A1-5c connections.

If node A2 is a single-mode node;

1. It will always have service continuity as long as it is within its parent gateway node X's coverage range through A2-1 connection.

2. In the event that $\mathrm{A} 2$ moves out of its parent gateway node's coverage range into network 2, it can have service continuity through:

a. Node R, a multi-mode terminal using A2-2a and A2-2b connections.

b. Node S, a multi-mode terminal using A2-3a and A2-3b connections. 
c. Node T, a multi-mode terminal using A2-4a and A2-4b connections.

d. Node U, a single-mode terminal using A2-5a and A2-5b connections.

3. If it happens that the only node that node A2 can link with is out of the range of all gateway nodes, service continuity can be supported by other available multihop node through all A2-6 connections.

From the scenario described, the focus of this research is on when the multihop nodes within the inter-working multihop wireless networks are out of their parent gateway node coverage and the continuity of access to service is provided through other single-mode or multi-mode nodes.

\section{Link availability}

A link is available to a node if there is a probability that its neighbor nodes are within at most the maximum transmission range that is sufficient for communication to be established between them. In a wireless network, the availability of a link between node pairs depends on the transmission range, the network's node density and the inter-nodal distance between them. Information about the inter-nodal distance allows an optimization of the link discovery process in inter-working multihop wireless networks. In addition a probabilistic model that provides knowledge about the availability of communication links could decrease the frequency of link failure that may be experienced during a routing process. Using the theory of homogenous Poisson Point Process (HPPP), we briefly present an analysis of inter-nodal distance distribution and the link availability model.

Theorem 1: For a HPPP in $\mathrm{R}^{2}$ (two-dimensional Euclidean plane), the probability that there are no nodes within a distance $x$ of an arbitrary node is $e^{-\lambda \pi x 2}$, where the parameter $\lambda$ is the mean number of nodes per unit area (node density) [21].

Let $\beta_{(\mathrm{T}, \mathrm{R})}$ be the distance between a T-node (source node) and its nearest neighbor (a potential R-node (destination node), $\mu_{\mathrm{Net}}$, the network's node density and $\mathrm{R}_{\mathrm{o}}$, a node's transmission range. Using theorem 1 , the probability that the distance between a T-node and its nearest neighbor is greater than the T- node's transmission range is:

$$
\operatorname{Pr}\left(\beta_{T, R}>R_{o}\right)=e^{-\mu_{N e t} \pi R_{o}^{2}} \quad \forall R_{o}>0
$$

The probability that the distance between a T-node and its nearest neighbor is less than its transmission range is:

$$
\operatorname{Pr}\left(\beta_{T, R} \leq R_{o}\right)=1-e^{-\mu_{N e t} \pi R_{o}{ }^{2}} \quad \forall R_{o}>0
$$

The cumulative distribution function (CDF) of the distance between any two randomly 
positioned nodes in the network is represented by (2). The probability that a communication link is available for any node is related to the link distance distribution [22]. A link is a 1-hop communication connection between node pairs and a route is a last mile multihop communication connection. Also, the probability that a last mile multihop communication path is available is related to the availability of the individual links that make up the path. As long as $\beta_{\mathrm{T}, \mathrm{R}} \leq \mathrm{R}_{\mathrm{O}}$, a link is available (exists) between any two arbitrary nodes. Therefore, the CDF of the link distance $\beta_{\mathrm{T}, \mathrm{R}}$ can be taken as the probability that at least a link is available for transmission. Thus, the availability of a link in a network is a function of $\mathrm{R}_{\mathrm{O}}, \beta_{\mathrm{T}, \mathrm{R}}$ and $\mu_{\mathrm{Net}}$. If $\mathrm{P}_{\text {link }}$ represents the availability of a 1-hop link, then, $\mathrm{P}_{\text {link }}$ is:

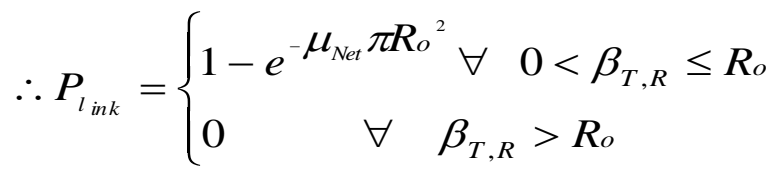

\section{Non impairment probability}

For resource optimization, we determine the non-impairment probability on available links. Non-impairment probability indicates the likelihood that the radio attributes of a link has the potential to satisfy the minimum requirement for successful communication over it. This requirement is defined by PHY/MAC layer parameters that ensure the proper functioning of a link. These include probability of bit error and signal to noise and interference ratio (SNIR). A link's SNIR $\left(\theta^{(l)}\right)$ is evaluated using (4). A transmission is said to be correctly decoded by an R-node on link $l$ if $\theta^{(l)}$ is not less than the SNIR threshold $\left(\theta^{\text {th }}\right)$ during a transmission [23].

$$
\theta^{(l)}=\frac{P_{l}^{r}}{P_{i n i}+P_{o}}=\frac{c P_{l}^{t}\left(\beta_{T, R}\right)^{-2}}{\sum_{k=1}^{S} c P^{t(k)}\left(\beta_{k, R}\right)^{-2}+P_{o}}
$$

With reference to link $l, \mathrm{P}_{l}^{\mathrm{r}}$ is the power received by the R-node, $\mathrm{P}_{l}^{\mathrm{t}}$ is the T-node's transmission power and $P_{o}$ is the noise power. The noise power $\left(P_{o}=F k T_{o} B\right.$ where $k$ is the Boltzman constant $\left(1.38 \times 10-23 \mathrm{~J} /{ }^{\circ} \mathrm{K} / \mathrm{Hz}\right), T_{o}$, the ambient temperature, B, the transmission bandwidth and $\mathrm{F}$ is the noise figure). $P_{i n i}$ is the cumulative of the interference power that an $\mathrm{R}$-node experiences from nodes concurrently transmitting with T-node. k-nodes (for $k=1$, $2, \ldots \infty)$ are potential interfering nodes while $S$ is the total number of simultaneously transmitting nodes that contributes to effective $P_{i n i}$. $P^{t(k)}$ is a k-node's transmission power 


\section{Macrothink}

and the distance between a k-node and the R-node is denoted by $\left(\beta_{k, R}\right)^{-2}$. As in fig. 3, an interference region $(\delta r)$ is defined for an R-node on link $l$. Nodes beyond $\delta r$ (i.e. B-nodes) do not to interfere with R-node's reception. N-nodes are non-interfering nodes and G-nodes are gateway nodes. The notation, $\mathrm{r}$ represents the T-node's transmission range $\left(R_{o}\right)$. A node will only interfere if the distance of the node to the R-node fulfils $r<\left|\beta_{k, R}\right| \leq r+\delta r$. According to [24] such nodes effectively contribute to the value of $P_{i n i}$ irrespective of the network topology or multiple-access technique. Normally, when a link is established between a T-node and R-node, the MAC technique will prohibit nearby nodes from transmitting simultaneously. The portion of the network occupied by these nearby nodes is directly related to the size of $r$ around the R- node [25].

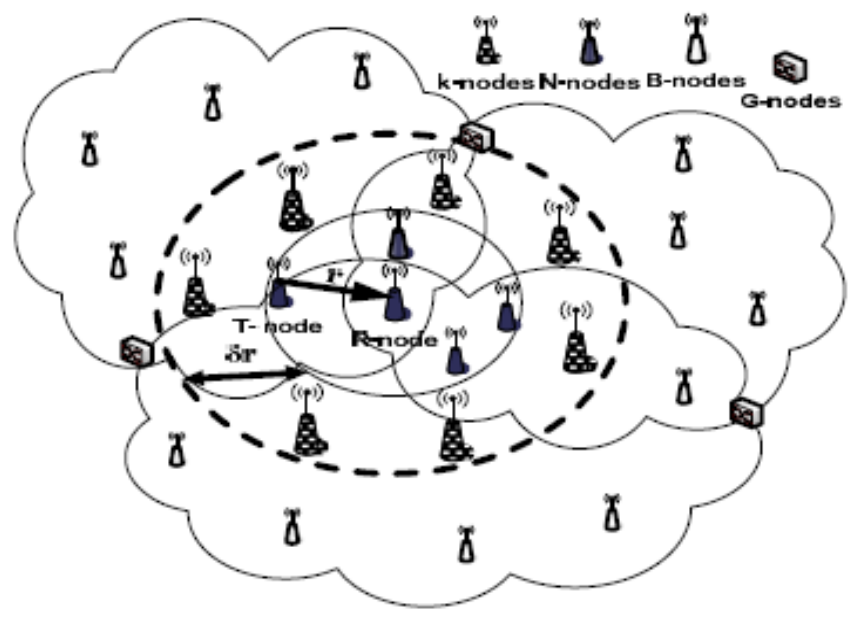

Figure 3. Illustration of a T-node to R-node transmission.

Theorem 2: $\mathrm{V} \lambda>\lambda_{\mathrm{i}}>0$, if a HPPP in $R^{2}$ with density $\lambda$ is randomly split into $N$ independent sub-processes with probabilities $P_{i}$ for $i=1 \ldots N$, such that, $P_{1}+P_{2} \ldots+P_{N}=1$, then these sub-processes are mutually independent HPPP with intensities $\lambda_{i}=\lambda P_{i}$ where $\lambda_{1} \lambda_{1}+\lambda_{2}+\ldots+$ $\lambda_{N}[26]$.

Using the splitting property of the HPPP in theorem 2, nodes in the inter-working network (with node density $\mu_{\mathrm{Net}}$ ) are sorted independently into k-nodes, N-nodes, and B-nodes. If the probability of a node being a k-node, $\mathrm{N}$-node or B-node is $P_{I}, P_{N}$, or $P_{B}$ respectively such that $P_{I}+P_{N}+P_{B}=1$, then these 3 types of nodes are mutually independent Poisson processes with densities: $\mu_{I}=P_{\mathbb{l}} \mu_{N e t}, \mu_{N}=P_{N} \mu_{N e t} \mu_{B}=P_{B} \mu_{N e t}$, where $\mu_{N e t}=\mu_{I}+\mu_{N}$ $+\mu_{B} \cdot \mu_{I}$ is the density of k-nodes, $\mu_{N}$ is the density of $\mathrm{N}$-nodes and $\mu_{B}$ is the density B-nodes. Now, the effective density of k-nodes can be obtained. Derivation of $P_{I}$ has been presented in [27]. Since $\beta_{k, R}$ values are Poisson random variables (r.v.) distributed in the $\delta r$ region, the expected value of $\beta_{k, R}$ in (4) can be estimated with (5). $\bar{\omega}$ is an approximate solution for the negative moment of $\beta_{k, R}$ using the Tiku's solution in [28]. 


$$
\begin{gathered}
E\left[\sum_{k=1}^{S}\left(\beta_{k, R}\right)^{-2}\right]=\sum_{k=1}^{S} E\left[\left(\beta_{k, R}\right)^{-2}\right]=\sum_{k=1}^{s} \varpi \\
\varpi \approx \frac{1}{\left(\mu_{I}-1\right)\left(\mu_{I}-2\right) \ldots \ldots \ldots\left(\mu_{I}-\tau\right)} .
\end{gathered}
$$

Therefore, in (4), $P_{\text {int }} \approx c P^{t[k 2)} \times S \times \bar{\omega}$, where, $S \approx P_{I} \times N_{\Omega}$ and $\mathbb{N}_{\Omega}$ is the total number of nodes in the network. Due to potential interference, communication may not be totally error-free; hence successful communication is specified in terms of an acceptable value for the probability of bit error $\left(\Phi^{(l)}\right)$ on link $l$. In order for an R-node to correctly receive a transmission, $\theta^{(l)} \geq \theta^{\text {th }}$. $\theta^{\text {th }}$ is a pre-set value that is used to ensure successful transmission in a network. Since $\theta^{\text {th }}$ is the threshold SNIR that is required for successful reception at the R-node, a transmission error can be declared if there is a probability that $\mathrm{E}\left(\theta^{(l)}\right)$ is below $\theta^{\text {th }}$ [27]. Therefore:

$$
\Phi^{(l)}=\operatorname{Pr}\left(E\left(\theta^{(l)}\right)<\theta^{\text {th }}\right)=1-\operatorname{Pr}\left(E\left(\theta^{(l)}\right) \geq \theta^{\text {th }}\right)
$$

Note that in order to maintain a certain SNIR threshold, there is an amount of interferers that can be supported. So, what is the maximum of the number of interfering nodes that can be within $\delta r$ such that a transmission error is declared if the number of interfering nodes exceeds this value? Let $S_{t h}$ represent the maximum number of interferers, whose distance to the R-node $\left(\beta_{k . R}\right)$ fulfils $r<\left|\beta_{k, R}\right| \leq r+\partial r$. Since unsuccessful transmission is declared when $E\left(\theta^{(l)}\right)<\theta^{t h}$, then probability of bit error occurs if $S>S_{t h}$.

There is an allowable $\mathrm{P}_{\text {ini }}$ that can sustain $\mathrm{E}\left(\theta^{(l)}\right) \geq \theta^{\text {th }}$ thus there is an amount of interferers that can be permitted within $\delta$ r for successful transmission to maintained. In order words, $\mathrm{E}\left(\theta^{(l)}\right)$ becomes less that $\theta^{\text {th }}$ as $S$ increases beyond $S_{t h}$ and $\Phi^{(l)}$ occurs on link $l$ when $\mathrm{E}\left(\theta^{(l)}\right)<\theta^{t h}$. Therefore,

$$
\begin{gathered}
\Phi^{(l)} \approx \operatorname{Pr}\left(S>S_{t h}\right)=1-\operatorname{Pr}\left(S \leq S_{t h}\right) \\
\Phi^{(l)} \approx 1-\sum_{k=0}^{S_{t h}} \frac{\left(\mu_{I} A_{I}\right)^{k}}{k !} e^{-\mu_{I} A_{I}}
\end{gathered}
$$

$\mu_{I}$ is the density of interfering nodes in $\delta r . A_{I}$ is the area of $\delta r$. Equation (9) approximates $\Phi^{(l)}$ as a function of the number of interfering nodes. Thus irrespective of the multiple access technique, only the effective density of interfering nodes is considered in 
estimating $\Phi^{(l)}$ [27]. The maximum number of interferers that can be supported to maintain the threshold for $\Phi^{(l)}$ is $S_{t h}$. Once $S$ exceeds $S_{t h}$, unsuccessful transmission is more likely to occur. A link's non-impairment probability, represented by $\Phi^{(l)^{\prime}}$ is given by the compliment of (9). Thus if the probability of bit error on a link is high, the non-impairment probability on that link is low and vice-versa. Non-impairment probability refers to the level of reliability of a link and the target is to utilize links with high non-impairment probability. A link with a high non-impairment probability is a reliable link.

\section{Connectivity model}

The routing process utilizes the connectivity model for determining the connectivity level, which is used as a routing metric. Most research works state that there is connectivity between any two nodes as long as they are within each other's transmission range. However, we have defined connectivity in inter-working multihop wireless networks as the probability that a wireless link is available and its non-impairment probability is high enough to guarantee a successful transmission over it. The probabilities (availability and non-impairment) are associated with a wireless link. They indicate the level of connectivity that a link can provide. Firstly, for a T-node, the probability that a link is available for it to transmit on is given by (3). The link may be a direct link to the intended destination node or it may be a link to intermediate nodes between the T-node and the intended destination. Secondly, an interference region is defined for the R-node (intended destination or intermediate node) on the link of interest. Thirdly, using PI, the density of interfering nodes $\left(\mu_{I}\right)$ and the total interference power $\left(P_{i n i}\right)$, which is used to evaluate $\mathrm{E}\left(\theta^{(l)}\right)$ on the link of interest are estimated. If $\mathrm{E}\left(\theta^{(l)}\right)<\theta^{\text {th }}$, then there is a likelihood that transmission errors will occur on that link. $\Phi^{(l)}$ is estimated and the non-impairment probability is derived with the compliment of (9). Finally, connectivity is estimated as.

$$
P_{\text {con }}=P_{\text {link }} *\left(1-\phi^{(l)}\right)
$$

Consider three inter-worked wireless multihop networks as shown in fig. 2; with 25 nodes (7 nodes, 8 nodes and 10 nodes respectively), covering an area of 25000 unit square. The transmission power of nodes is $10 \mathrm{~mW}$ and transmission range is 100 unit. $G t$ and $G r$, the transmitter and receiver gains are assumed to be equal to $1, \mathrm{~L}_{\mathrm{f}}=1$, and $\theta^{\text {th }}$ is $6 \mathrm{~dB}$. The interference region is 1000 unit square. Fig. 3 shows the analytical evaluation of $\mathrm{P}_{\text {con }}$ on link 


\section{Macrothink}

$l$ between a source node and a receiver node as $\mu_{N e t}$ increases. At very low $\mu_{N e t}$ and the given transmission power, $P_{\text {link }}$ is very low and the T-node can barely reach other nodes. As $\mu_{N e t}$ increases gradually, a reasonable number of links become available for the T-node and $\Phi^{(l)^{\prime}}$ on such links is high. However, the $P_{c o n}$ level on these links is quite low. At high values of $\mu_{\text {Net }}, P_{\text {link }}$ increases and though $\Phi^{(l)^{\prime}}$ reduces yet $P_{\text {con }}$ level rises. The reduction in $\Phi^{(l)^{\prime}}$ is due to the rise in $\mu_{I}$. However, the rise in $\mu_{I}$ does not reduce the $P_{c o n}$ level because the amount of interfering nodes $(S)$ is still keeping $\mathrm{E}\left(\theta^{(l)}\right)$ less than or equal to the threshold value and thus $\Phi^{(l)}$ is still good enough for useful transmission. At higher values of $\mu_{N e t}, P_{\text {link }}$ continues to rise and $\Phi^{(l)^{\prime}}$ reduces still. At this stage, $P_{c o n}$ begins to decline because the rise in $\mu_{N e t}$, causes the interferers to surpass the tolerable amount. Therefore, connectivity cannot be guaranteed any longer at this stage. Generally, from literature, connectivity for a T-node is evaluated based on either; 1) the amount of potential R-nodes it can reach (i.e. the greater the number of nodes within its reach, the higher the connectivity) [29] or 2) the level of interference on the link between itself and potential R-nodes (the lower the interference, the higher the connectivity).

However, in this research we observed that there is a trade-off between $P_{\text {link }}$ and $\Phi^{(l)^{\prime}}$. Fig.4 illustrates this trade-off as follows: At low $\mu_{N e t}, \Phi^{(l)^{\prime}}$ is high but very few potential $\mathrm{R}$-nodes can be reached ( $\mathrm{P}_{\text {link }}$ is low). At high $\mu_{\text {Net }}$, more potential R-nodes can be reached ( $\mathrm{P}_{\text {link }}$ is high), however, $\Phi^{(l)^{\prime}}$ is low.

There is an issue with inclining directly to any of the two notions mentioned because low $P_{\text {link }}$ may lead to difficulty in finding potential R-nodes while high $P_{\text {link }}$ may lead to an unreliable network. Our connectivity model solves this trade-off issue by combining $P_{\text {link }}$ and $\Phi^{(l)^{\prime}}$ in the evaluation of connectivity. Focusing on left portion of fig. 3, the model evaluates connectivity as follows:

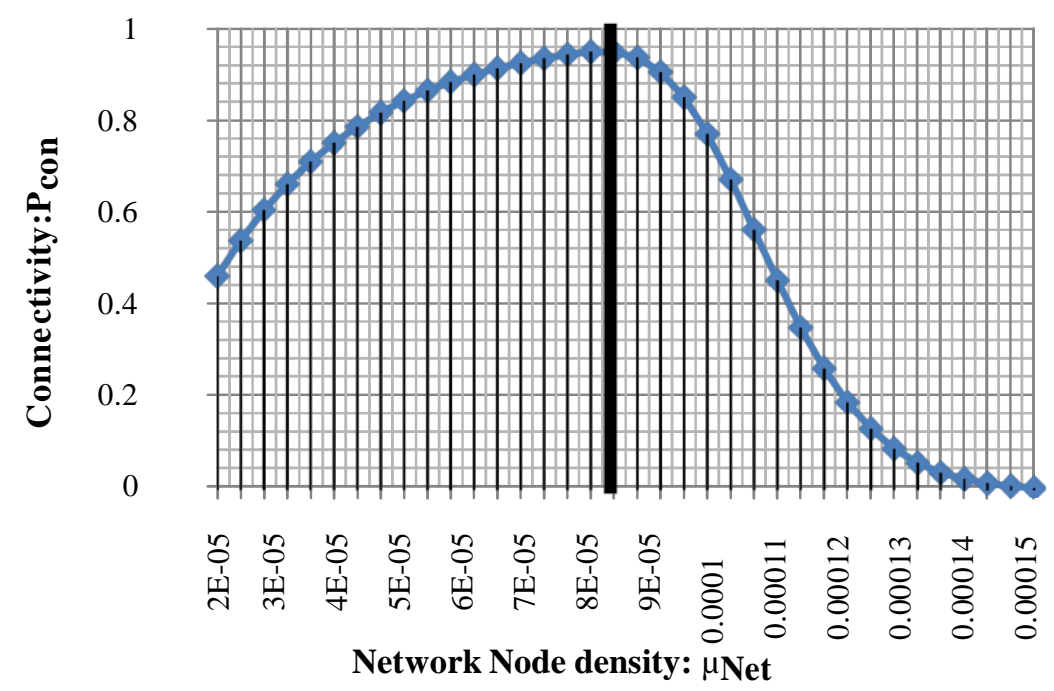

Figure 4. Connectivity vs Network node density.

- At very low $\mu_{N e t}$, connectivity $\left(P_{c o n}\right)$ is taken as nonexistent as the T-node can 
barely reach other nodes.

- At low $P_{\text {link }}$, though $\Phi^{(l)^{\prime}}$ is high, $P_{\text {con }}$ on a link is termed low.

- At high $P_{\text {link }}, \Phi^{(l)^{\prime}}$ is favorable and $P_{\text {con }}$ is optimal.

It is desirable to route traffic through links with optimal connectivity. Note that

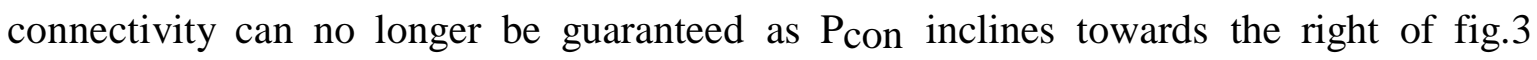
because $\Phi^{(l)^{\prime}}$ becomes unfavorable due to the high interference level caused by high node density.

In summary, the first step in the TE framework is the calculation of $P_{\text {link }}$ in order to identify 1-hop nodes. For all available links, the second step is to obtain $P_{I}$ at the R-node on the link, the SNIR and the probability of bit error. Since a change in link quality levels affects connectivity between nodes, these parameters allow the consideration of link quality in the computation of the routing metric $\left(P_{c o n}\right)$. The third step is to evaluate $\Phi(l)^{\prime}$ on each potentially available link. The fourth step is to use $P_{\text {link }}$ and $\Phi(l)^{\prime}$ to obtain $P_{\text {con }}$ on each link. The routing process chooses links that can provide optimal level of $P_{c o n} . P_{c o n}$ levels estimated over individual links are multiplied to get the overall $P_{c o n}$ for potential routes.

\section{Analysis of simulation performance of the framework}

For the simulation of the framework, the NS-Miracle-1.2.2 and NS2.34 versions were installed on Ubuntu 9.10. The network scenario of fig.1 was simulated. Specifically, nodes are either single or dual mode nodes with IEEE 802.11 a or IEEE 802.11 b or both. At the time of this simulation, the IEEE 802.11 standards were the only multihop technology on the NS-Miracle simulator. Also, only the PHY/MAC layer configurations of IEEE $802.11 \mathrm{~b}$ are on NS2.34, so for the purpose of this research, configurations for IEEE 802.11a were added to the simulator. To ensure the proper functioning of the MAC layer, the packet error rate (PER) information for IEEE 802.11a at applicable data rates adapted from [30] was incorporated. The MAC codes were modified to create the connectivity aware MAC, which allows the dynamic computation of the probability of bit error. For the routing process, codes were added to obtain $\mathrm{P}_{\text {link }}, \Phi^{(l)^{\prime}}$ and $P_{\text {con }}$. We simulated the framework's routing process employed by the protocol we termed AODV-UU-CL and another routing process that employs hop-count (AODV-UU-HP), which is on NS-miracle. This allowed a comparison of the performance of the connectivity metric and the hop count metric. The simulation metrics considered are:

a) Good throughput: total number of application layer packets successfully transmitted in the network per second.

b) Delay: time (in seconds) it takes data packets to reach their destinations.

c) Packets Lost: number of packets that were lost.

d) Packet delivery ratio: ratio of the number of data packets successfully received by 


\section{Macrothink}

destination nodes to the total number of data packets sent by source nodes. Using both single and multi mode nodes, simulations were performed by:

1) Varying the number of nodes (node density $\left(\mu_{\mathrm{Net}}\right)$ ) from 20 to 180 with increments of 20 , while the number of nodes transmitting at a time remains constant at 12 nodes.

2) Varying the simultaneously transmitting nodes (interfering node density $\left(\left(\mu_{I}\right)\right)$ from 2 to 20 with increments of 2 , while the number of nodes in the network remains 60 nodes. Nodes locations are Poisson distributed. Total simulation time was 450 secs, the distance between nodes was $150 \mathrm{~m}$ and the transmitting power of nodes was $10 \mathrm{~mW}$.

\subsection{Simulation results with AODV-UU-CL and AODV-UU-HP}

This sub-section provides the comparison of the simulation results of the AODV-UU-CL and the AODV-UU-HP.

a) Good-put: Good-put is better with AODV-UU-CL as the number of nodes increases even though the performance of the two metrics fluctuates as shown in fig.5. AODV-UU-CL keeps track of network link conditions in order to choose routes with optimal connectivity during the routing process, and thus is able to transmit more packets successfully than AODV-UU-HP, which is oblivious to link conditions during its routing. Thus, in an inter-working multihop wireless network scenario, AODV-UU-CL can maintain ubiquitous service continuity for nodes.

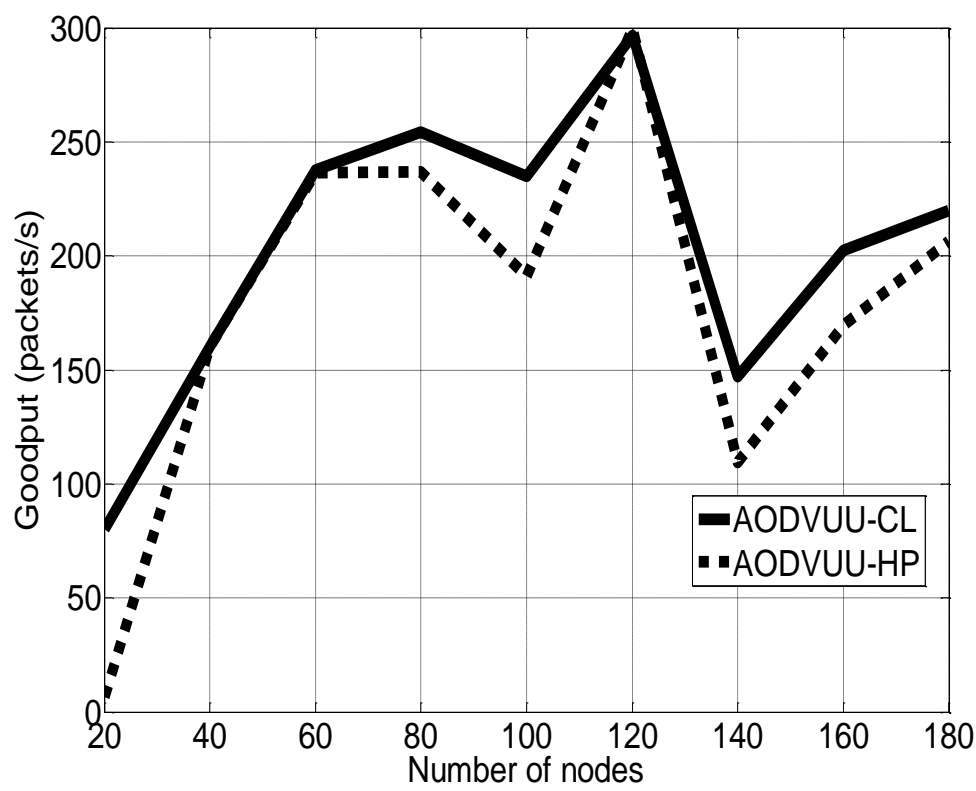

Figure 5 Good-put vs number of nodes

In fig.6, both metrics tend to choose equally good links for transmission when the number of nodes transmitting at a time is low. However, as the transmitting nodes increase 


\section{Macrothink}

beyond 14nodes, AODV-UU-CL is able to transmitting more packets with success. At this stage, the good-put of AODV-UU-HP starts to decline as it chooses routes without considering the network link quality. Fig.6 shows that though having more simultaneously transmitting nodes reduces $\Phi^{(l)^{\prime}}$ on links, yet AODV-UU-CL has the ability to avoid impaired links than AODV-UU-HP. A low hop-count route does not necessarily indicate reliable transmission as links may have been chosen around the interfering nodes.

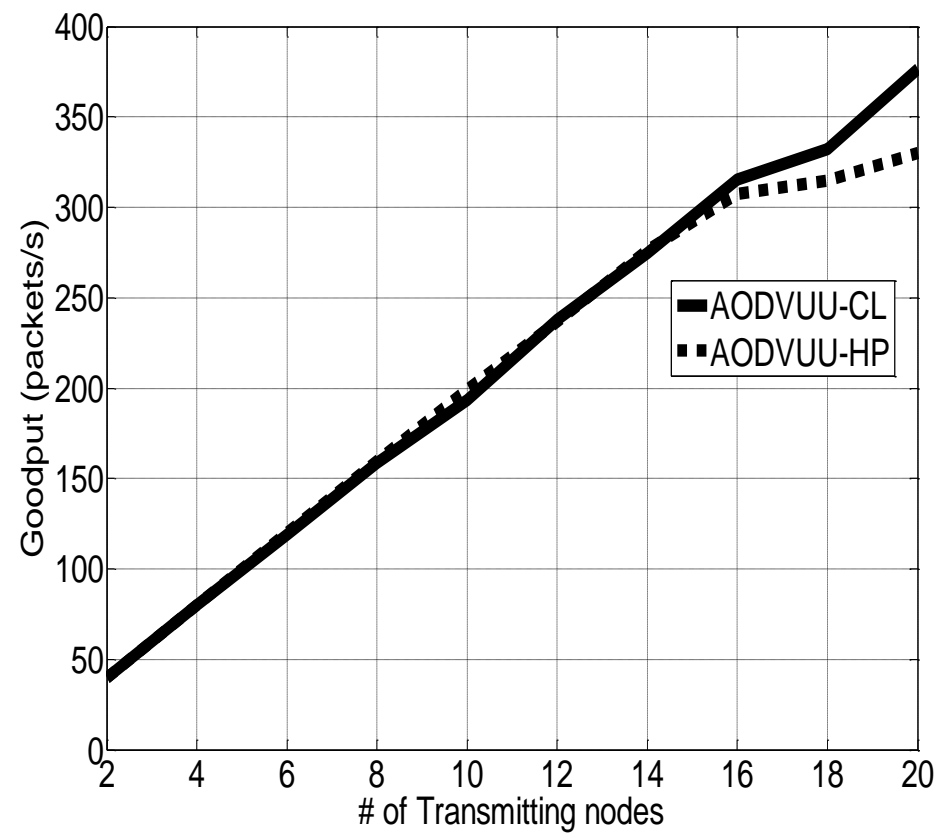

Figure 6 Good-put vs \# of transmitting nodes

b) Packet delivery ratio/percentage: In fig. 7, at low network node density, the performance of both metrics is comparable but a distinction is seen as the number of nodes increases. The delivery ratio is higher with AODV-UU-CL as node density increases, this is also because AODV-UU-CL is careful to avoid links that are impaired, but AODV-UU-HP does not evade such links. In fig. 8, the delivery ratio for both metrics begins to decrease after 14 transmitting nodes, but the delivery ratio for AODV-UU-CL remains higher than that of AODV-UU-HP after 14nodes. 


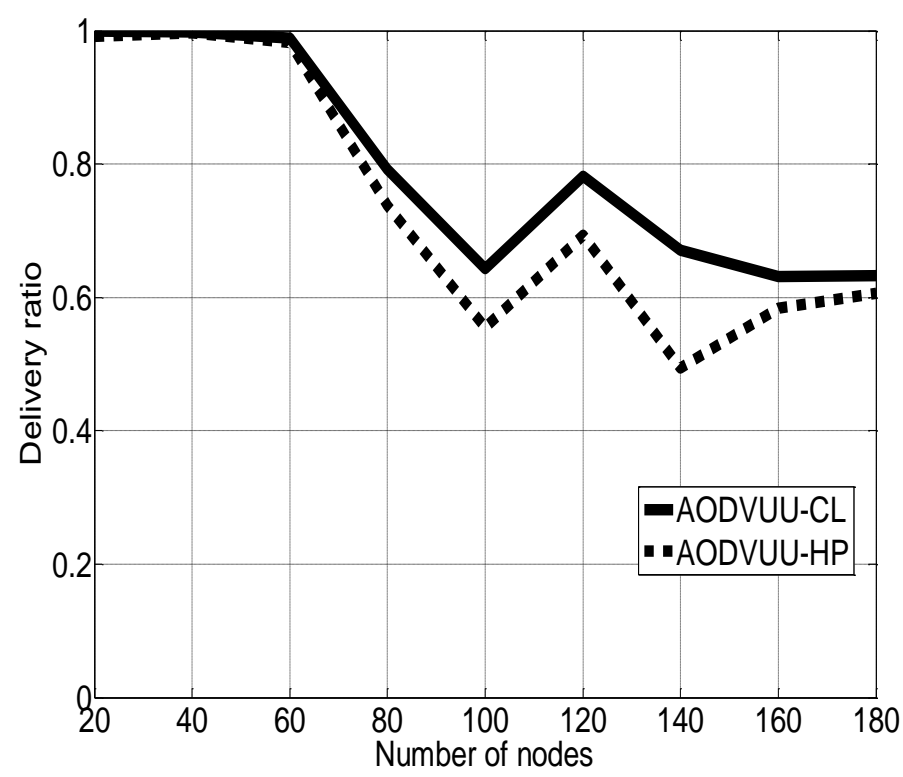

Figure 7 Delivery ratio vs \# of nodes

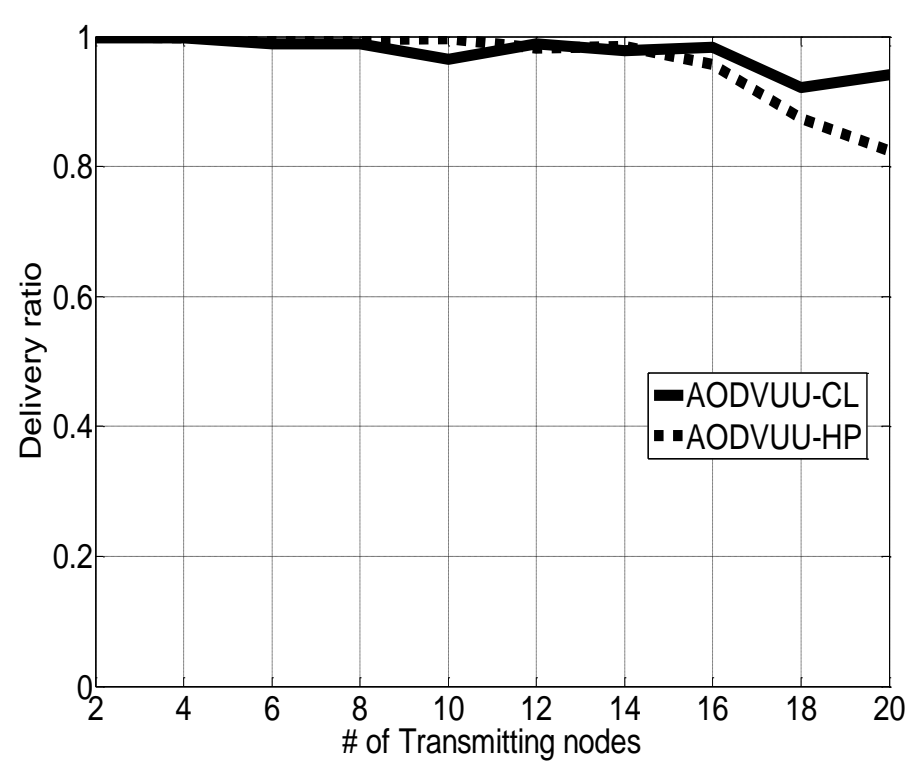

Figure 8 Delivery ratio vs \# of transmitting nodes

c) Packets Lost: In fig. 9, more packets are increasingly lost with AODV-UU-HP as the number of nodes increases beyond 60 nodes in the inter-working network and as the number of transmitting nodes rise above 10 nodes in fig. 10. With AODV- UU-CL, the number of packets lost increases as the number of nodes increases but it remains significantly lower than the amount lost by AODV-UU-HP beyond 60nodes and 120nodes in the network. Initially, in fig. 10, a negligibly higher number of packets are lost by AODV-UU-CL at low number of transmitting nodes, but a great distinction in AODV-UU-CL's performance can be seen after 14 transmitting nodes. With more than 14 simultaneously transmitting nodes, AODV-UU-CL losses lesser packets than AODV-UU-HP. 


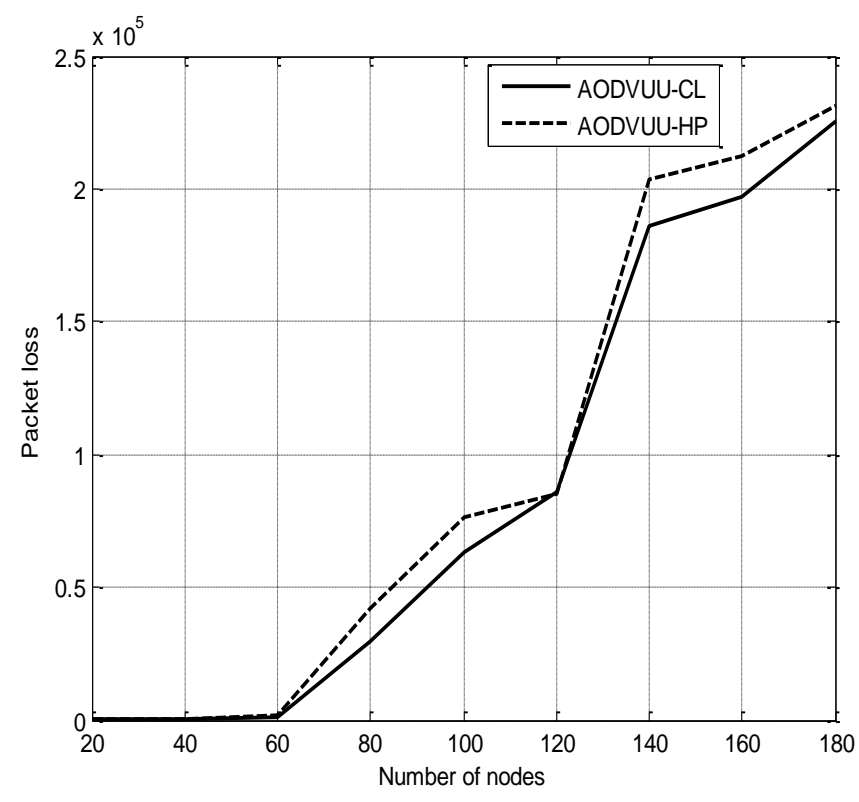

Figure 9 Packet lost vs number of nodes

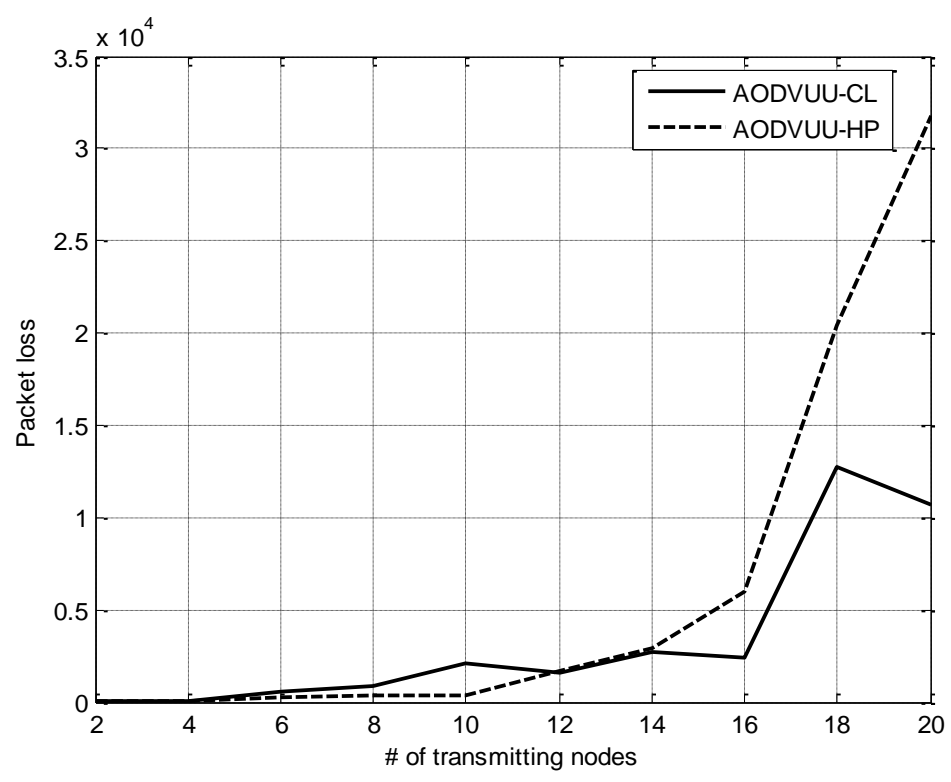

Figure 10 Packet lost vs \# of transmitting nodes

d) Delay: From fig. 11, the delay performance of both metrics fluctuates as the number of nodes increases beyond 60 nodes. Initially, the delay performance of AODV-UU-CL is slightly lower than that of AODV-UU-HP until after 60 nodes. However, as the number of nodes increases beyond 120 nodes, more nodes are available $\left(P_{\text {link }}\right.$ is high) and because $\Phi^{(l)^{\prime}}$ is favorable, AODV-UU-CL is able to choose links with optimal connectivity, thus averagely reducing delay during the transmission of packets. AODV-UU-HP chooses low hop-count route irrespective of the quality of the link and its performance fluctuates with higher values of delay on the average as compared to AODV-UU-CL. In fig. 12, as the number of 


\section{Macrothink}

transmitting nodes increase, delay also fluctuates with both AODV-UU-HP and AODV-UU-CL. AODV-UU-HP begins to highly decline in performance, but the performance of AODV-UU-CL does not decline as much as AODV-UU-HP. This is because the connectivity metric is able to choose links that provides optimal connectivity levels.

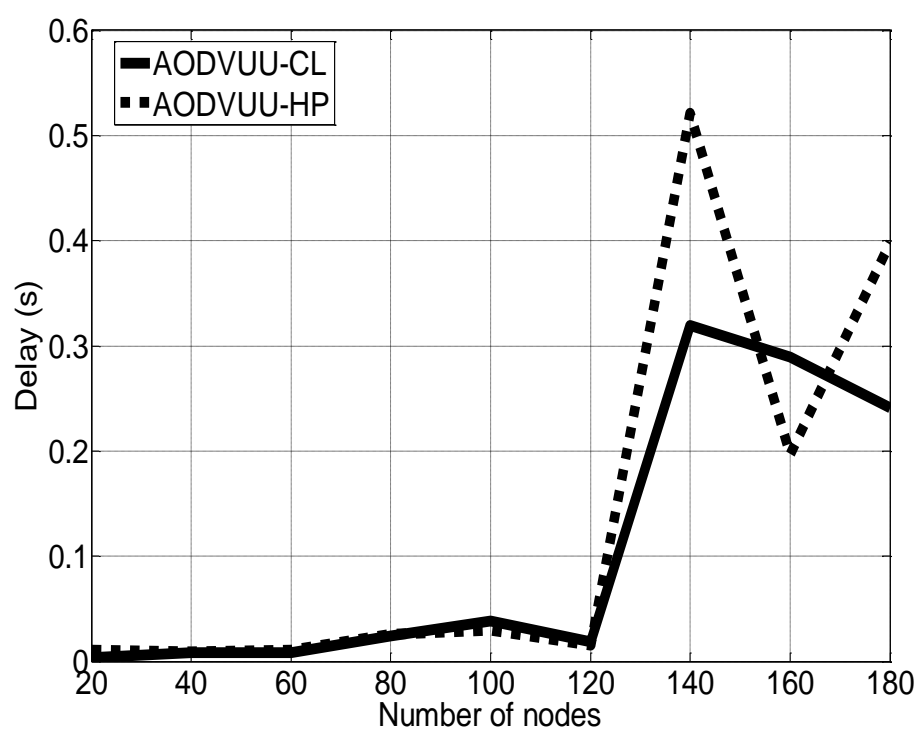

Figure 11 Delay vs number of nodes

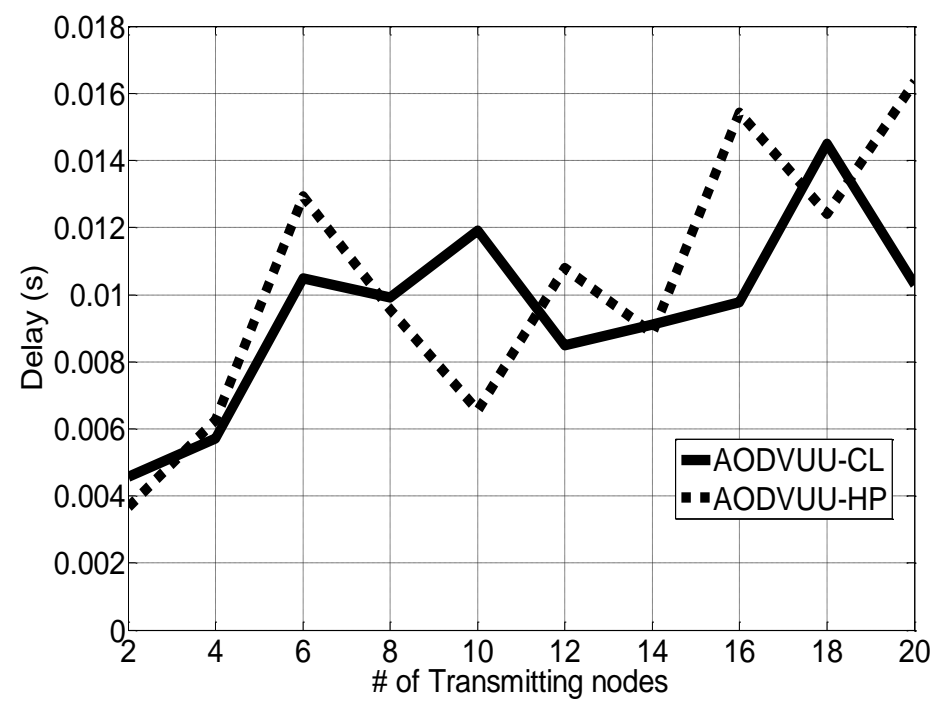

Figure 12 Delay vs \# of transmitting nodes

Further simulation results obtained to observe the delay metric are shown in fig.13 and 14. The distance between nodes and the transmission power of nodes were varied while the number of nodes and number of transmitting nodes in the network remained fixed at 60 nodes and 12 nodes respectively. The delay performance of both metrics under varying nodal distances and transmission power also fluctuates. However, from fig. 12 and 13, 


\section{Macrothink}

AODV-UU-CL improves the delay experienced by nodes in the network more than AODV-UU-HP. Generally for AODVUU-CL and AODVUU-HP, an increase in the distance between nodes decreases the hop distance between nodes (i.e. shorter distances means more hops and longer distances means lesser number of hops), thus delay reduces as distance increases. At higher transmission power, delay is reduced.

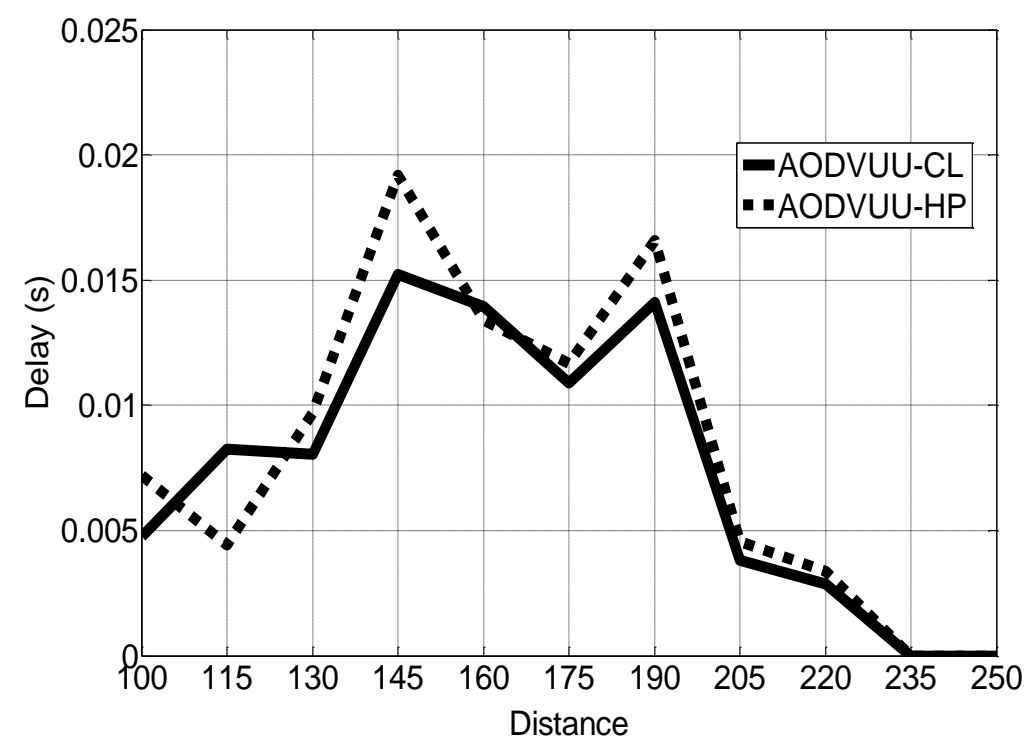

Figure 13 Delay vs Distance

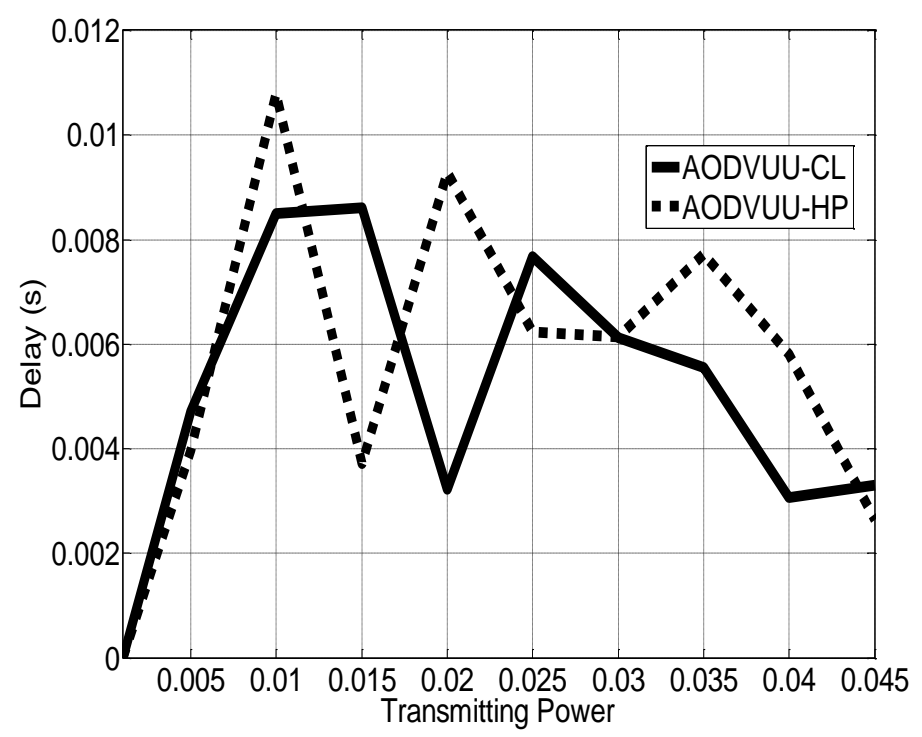

Figure 14 Delay vs Transmitting power

In fig. 5 to 12, the distinction in the performance of AODV-UU-CL and AODV-UU-HP is clearer as the network node density (number of nodes within the network) and the interfering node density (number of transmission nodes) becomes increasingly high. In the interworking multihop network scenario, as the network gets denser, (i.e. when the number of nodes in the 
network is increased), AODV-UU-CL exhibited a better performance than AODV-UU-HP. In addition, when the number of transmitting nodes is increased (which increases the number of interfering nodes), AODV-UU-CL performs better in terms of good-put, packet delivery ratio, number of packet lost and delay.

\section{Conclusion}

The findings related to the performance of the proposed framework indicate that with an increase in the network node density and interfering node density, which degrades network performance, the framework is able to achieve improved QoS at the traffic level. It successfully delivers more packets to the application layer. It is able to reduce the number of packets lost during transmission and the percentage of packets delivered also increases. Though the delay performance fluctuates as the network gets more congested with nodes (interfering nodes), it still provided lower delay values in comparison with the framework that employs hop-count. The improved performance can be attributed to choosing link with optimal connectivity to make up a multi-hop route. The overall performance shows that it can be used to ensure better and reliable ubiquitous network service access and continuity in an inter-working multi-hop wireless networks with heterogeneous mobile terminals (single-mode and multi-mode nodes).

With regards to future work, since only link metrics were considered in this research, the authors have considered the inclusion of node metrics such as energy or power levels and memory or workload in the connectivity model. In addition, nodes may experience different communication conditions when they are within the coverage range of their parent gateway node and when they are out of range. Thus, it will be interesting to enhance nodes with multiple routing mechanisms so that they can adaptively switch routing mechanisms depending on the network scenario (i.e. if they are in or out of their gateway node's range). They can use an applicable routing process when they are within the range of their gateway nodes and use the proposed routing process when they are outside their gateway node's coverage (i.e. using other nodes to relay their traffic).

\section{References}

[1] M. Shin, J. Ma, A. Mishra, and W.A. Arbaugh, "Wireless Network Security and Interworking," Proceedings of the IEEE, vol.94, Issue 2, pp.455 - 466, Feb. 2006. http://dx.doi.org/10.1109/JPROC.2005.862322.

[2] H.Y. Hsieh and R. Sivakumar, "Internetworking WWANs and WLANs in Next Generation Wireless Data Networks," International Conference on Third Generation Wireless and Beyond (3Gwireless), San Francisco, CA, USA, pp. 334-339, May 2002. http://dx.doi.org/10.1.1.4.7694.

[3] R. Ferrus, O. Sallent, and R. Agusti, "Interworking in heterogeneous wireless Networks: comprehensive framework and Future trends," IEEE Wireless Communications, vol.17 Issue 2, pp 22-31, April 2010, http://dx.doi.org/10.1109/MWC.2010.5450657. 
[4] M. Dohler, D. E. Meddour, S.M. Senouci, and A. Saadani, "Cooperation in 4G - Hype or Ripe?” IEEE Technology and Society Magazine, vol. 27 Issue 1, pp13-17, March 2008. http://dx.doi.org/10.1109/MTS.2008.918035.

[5] 3GPP TS 23.234, “3GPP system to WLAN interworking," Version.6.5.0, June 2005.

[6] M. Buddihikot, G. Chandranmenon, S. Han, Y. Lee, S. Miller, and L. Salgarelli, "Design and implementation of a WLAN/CDMA2000 interworking architecture," IEEE $\begin{array}{lllll}\text { Communications } \quad \text { Magazine, } & \text { vol.41, } & \text { Issue } & 11, & \end{array}$ http://dx.doi.org/10.1109/MCOM.2003.1244928.

[7] S. W. Peters and R.W. Heath, "The future of WiMAX: multihop relaying with IEEE 802.16j," IEEE Communications Magazine, vol.47, Issue 1, pp 104-111, January 2009. http://dx.doi.org/10.1109/MCOM.2009.4752686.

[8] S. Frattasi, H. Fathi, F.H.P. Fitzek, R. Prasad, and M. D. Katz, "Defining 4G Technology from the User's Perspective," IEEE Network, vol. 20 Issue 1, Jan.- Feb. 2006. http://dx.doi.org/10.1109/MNET.2006.1580917.

[9] S. Saraf, "Co-operative Networks for 4G," 23rd January, 2010, Available at: http://www-scf.usc/edu/ ssaraf/ EE555.pdf on.

[10]X. J. Li, B.-C. Seet, P. Han and J.Chong "Multihop cellular networks: Technology and economics," Computer Networks, vol. 52, pp. 1825-1837, 2008. http://dx.doi.org/10.1016/j.comnet.2008.01.019.

[11]C. Politis, T. Oda, S. Dixit, A. Schieder, H.Y. Lach, M.I. Smirnov, S. Uskela, and R. Tafazolli, "Cooperative Networks for the Future Wireless World," IEEE Communications Magazine, vol. 42, Issue 9, pp. 70- 79, ${ }^{2}$ Sept. 2004. http://dx.doi.org/10.1109/MCOM.2004.1336723.

[12]A. Ribeiro, T. Luo, N. Sidiropoulos and G. Giannakis, "Modeling and optimization of stochastic routing for wireless multihop networks," Proc. IEEE International Conference on Computer Communication, May 6-12 2000, Anchorage AK, pp. 1748-1756. http://dx.doi.org/10.1109/INFCOM.2007.204.

[13]IEEE Std 802.16j-2009, "IEEE Standard for Local and Metropolitan Area Networks Part 16: Air Interface for Broadband Wireless Access Systems -Amendment 1: Multi-hop Relay Specification,” 12 June 2009.

[14]3rd Generation Partnership Project; Technical Specification Group Radio Access Network; Opportunity Driven Multiple Access (3G TR 25.924 version 1.0.0), 1999, available at: http://www.3gpp.org/ on 14th Sept, 2009.

[15]G. Calcev and J. Bonta, "OFDMA Cellular Networks with Opportunistic Two-Hop Relays," EURASIP Journal on Wireless Communications and Networking, Volume 2009, Article ID 702659, pp. 2-9. http://dx.doi.org/10.1155/2009/702659.

[16] M. J. Lee, J. Zheng, Y. B. Ko and D. M. Shrestha, "Emerging standards for Wireless Mesh Technology," IEEE Wireless Communications, pp 56 - 63, April 2006, http://dx.doi.org/10.1109/MWC.2006.1632481.

[17]D. Cavalcanti, D. Agrawal, C. Cordeiro, B. Xie and A. Kumar, "Issues in integrating cellular networks, WLANs, and MANETs: A Futuristic Heterogeneous wireless network," IEEE Wireless Communications, pp. 30-41, June 2005, http://dx.doi.org/10.1109/MWC.2005.1452852. 
[18] "Overview of Recommendations on traffic engineering" ITU-T Recommendation E.490.1, Jan. 2003.

[19] O. Salami, H. A. Chan, and, A. Bagula, "Multi-layer Resource Optimization and QoS guarantee for Inter-working Wireless Networks," In proceedings of the 3rd International Conference on Broadband Communications, Information Technology and Biomedical Applications, November 23-26, 2008, pp. 120-123, http://dx.doi.org/10.1109/BROADCOM.2008.46.

[20]A. Henrik and G. Anders, "Traffic engineering in ambient networks: challenges and approaches," Proceedings of the Second Swedish National Computer Networking Workshop, 23-24 Nov 2004, Karlstad, Sweden, 2004.

[21]N. A. C. Cressie, "Statistics for Spatial Data," John Wiley and Sons, 1991.

[22]L. E. Miller. "Distribution of link distances in a wireless network." Journal of Research of the National Institute of Standards and Technology, 106(2), pp 401-412, 2001.

[23] P. Gupta and P. R. Kumar, "The Capacity of Wireless Networks," IEEE Transactions on Information Theory, vol. 46, No.2, pp. 388-404, 2000. http://dx.doi.org/10.1109/18.825799. [24] M. Win, P. Pinto and L. Shepp, "A Mathematical Theory of Network Interference and Its Applications," IEEE transaction, vol.97, 2009. http://dx.doi.org/10.1109/JPROC.2008.2008764.

[25]R. Hekmat and X. An, "Relation between Interference and Neighbor Attachment Policies in Ad-hoc and Sensor Networks," International Journal of Hybrid Information Technology, vol. 1, No. 2, 2008.

[26] N. Hohn, D. Veitch and T. Ye, "Splitting and merging of packet traffic: measurement and modeling," Performance Evaluation, vol. 62, Issue 1-4, pp164-177, 2005.

[27]O. Salami, A. Bagula and H. A. Chan. "Framework for Link Reliability in Interworking Multihop Wireless Networks", Mathematical and Computer Modeling, An International Journal, by Elsevier, Vol.53 (2011) pp. 2219-2228, http://dx.doi.org/ 10.1016/j.mcm.2010.08.027.

[28] M. L. Tiku, "A note on the negative moments of a truncated Poisson variate," Journal of American Statistical Association vol.59, No 308, pp 1220-1224, 1964.

[29]Q. Ling and Z. Tian, "Minimum Node Degree and k-Connectivity of a Wireless Multihop Network in Bounded Area," IEEE GLOBECOM, pp 1296-1301, 2007. http://dx.doi.org/10.1109/GLOCOM.2007.249.

[30]F. A. Tobagi, "Interactions between the Physical Layer and Upper Layers in Wireless Networks: The devil is in the details," a presentation at "Broadnets 2006", San Jose, 4th Oct. 2006, available at: http://www.broadnets.org/2006/downloads/invited-talks/Tobagi.pdf. [Last accessed January 3, 2012].

\section{Copyright Disclaimer}

Copyright reserved by the author(s).

This article is an open-access article distributed under the terms and conditions of the Creative Commons Attribution license (http://creativecommons.org/licenses/by/3.0/). 